\title{
Original
}

\section{Pilot Study of Bone Augmentation in Rat Calvaria Using Silicone Molds with Recombinant Human Bone Morphogenetic Protein-2-containing Atelocollagen Sponge}

\author{
Kenji Narita $^{* 1,2)}$, Mitsuo OdA ${ }^{1,2)}$, Mitsuori MayaharA ${ }^{1,3)}$, \\ Kazuhiro UmeharA ${ }^{2)}$, Masatoshi UmeharA ${ }^{2)}$, Eisaku IMAMUrA ${ }^{1)}$, \\ Ryuta KATAOKA ${ }^{3)}$, Hiroto KIMURA ${ }^{2,4)}$ and Masanori NAKAMURA ${ }^{1)}$
}

\begin{abstract}
Bone augmentation is required for cases of highly absorbed alveolar ridge in the placement of dental implants. Various biomaterials and/or growth factors have been used to induce new bone formation; however, it remains difficult to obtain the required bone shape. In this study, we used cylindrical silicone molds coupled with recombinant human bone morphogenetic protein (rhBMP) to induce a specifically shaped bone. The cylindrical mold was made of silicone rubber impression material with a $10 \mathrm{~mm}$ outer diameter, $5 \mathrm{~mm}$ inner diameter, and $5 \mathrm{~mm}$ height. RhBMP-2 was applied using an atelocollagen sponge placed into the silicone mold; it was then implanted into the parietal subperiosteal region of 8-week-old male Wistar rats. Histological and micro-computed tomography (micro-CT) analyses were performed to detect new bone formation at 2,4 , and 8 weeks after the surgery. Micro-CT analysis indicated a disc-shaped bone formation adjacent to the periosteum at 2 weeks after surgery. Newly formed bone was also detected near the parietal bone at 4 weeks. At 8 weeks, an almost cylindrical bone was formed in the silicone mold, and histological observation confirmed that the newly formed bone completely adhered to the host parietal bone. Hematopoietic bone marrow was also detected in the newly formed bone. A specifically made silicone mold might provide the scaffold required to induce a specific shape of newly formed bone under the combined induction of rhBMP.
\end{abstract}

Key words : recombinant human bone morphogenetic protein-2 (rhBMP-2), silicone mold, atelocollagen sponge, onlay grafting

\section{Introduction}

In oral and maxillofacial surgery, bone grafting is used to recover bone defects due to malformation, cysts, or tumors. Recently, sinus lifting has been selected to augment bone volume for dental implant installation in cases of highly atrophic alveolar ridge, particularly

1) Department of Oral Anatomy and Developmental Biology, Showa University School of Dentistry, 1-5-8 Hatanodai, Shinagawa-ku, Tokyo 142-8555, Japan.

2) Society of Aomori Implant Research.

3) Department of Dental Education, Showa University School of Dentistry.

4) Department of Dentistry and Oral Surgery, Hirosaki University Graduate School of Medicine.

* To whom corresponding should be addressed. 
in the maxillary molar region ${ }^{1-5)}$. Autogenous bone grafting has been performed in many of these cases as the long-standing "gold standard" ${ }^{6}$, however, this method of grafting requires another operation field for bone harvesting, such as the iliac region, potentially increasing the risk of morbidity ${ }^{7-10)}$. Achieving adequate osseointegration and stable function after the implant placement requires that the newly formed bone is well incorporated into the original host bone, it is of suitable quantity, and also of the desired shape. To this end, various biomaterials and/ or growth factors have been investigated for many years as alternative clinical methods to bone grafting $^{11-15)}$.

Urist $^{16)}$ first reported the concept of bone morphogenetic protein (BMP) as an osteoinductive factor; Wozney et $a l^{17)}$ and Wang et $a l^{18)}$ were the first to purify and clone this protein. Subsequently, many studies have applied BMP effectively clinically, and atelocollagen is now an accepted and potent carrier of BMP for the induction of newly formed bone ${ }^{19-22}$.

Alam et $a l^{23)}$ and Hosoya et $a l^{24)}$ recently induced bone formation to a desired shape using BMP / collagen in silicone molds. Herein, we aimed to similarly obtain newly formed bone to a specified shape and quality for implant placement using BMP and silicone molds in rat calvaria.

\section{Materials and methods}

\section{Preparation of implant materials}

A silicone rubber material (vinyl polysiloxane impression material, GC Dental Products Co., Tokyo, Japan) was used to make the molds for inducing bone tissue formation. A cylindrical silicone mold (10 $\mathrm{mm}$ outer diameter, $5 \mathrm{~mm}$ height) was made using a made-to-order template. The inner diameter of the mold was then standardized to $5 \mathrm{~mm}$ (Fig. 1). The mold was sterilized with ethylene oxide gas, and an atelocollagen sponge (TERUPLUG ${ }^{\circledR}$, Olympus Terumo Biomaterial Co., Tokyo, Japan) was cut so as to just fill the inner space of the mold. Under aseptic conditions, $10 \mu \mathrm{g}$ of rhBMP-2 (PeproTech, Inc., Rocky Hill, NJ, USA) dissolved in $10 \mu \mathrm{l}$ of physiological saline was added to the atelocollagen sponge. In the control group, only physiological saline was applied.

\section{Animals and surgical procedures}

The experimental protocol used was reviewed and approved by the Animal Care Committees of Showa University and Hirosaki University. Eight-week-old male Wistar rats (weight 250-300 g) were divided into two groups: BMP-treated group $(\mathrm{N}=15)$ and control group $(\mathrm{N}=6)$. Rats were anesthetized with an intraperitoneal injection of sodium pentobarbital $(2.5 \mathrm{mg}$ per $100 \mathrm{~g}$ body weight). All surgeries were performed under sterile conditions.

The skin of the head was shaved and then disinfected with ethanol and iodine. A curved horizontal incision $(1.5 \mathrm{~cm})$ was made in the parietal region between the ears and the periosteum was elevated to form a pocket and expose the bone surface. On the occasion of elevating the periosteum, a $1.0 \mathrm{~cm}$ periosteal incision was made at the posterior border of the parietal bone, and then the periosteum was ablated forward to make a subperiosteal pocket large enough to fit the implanted silicone mold. A silicone mold containing BMP/collagen was 

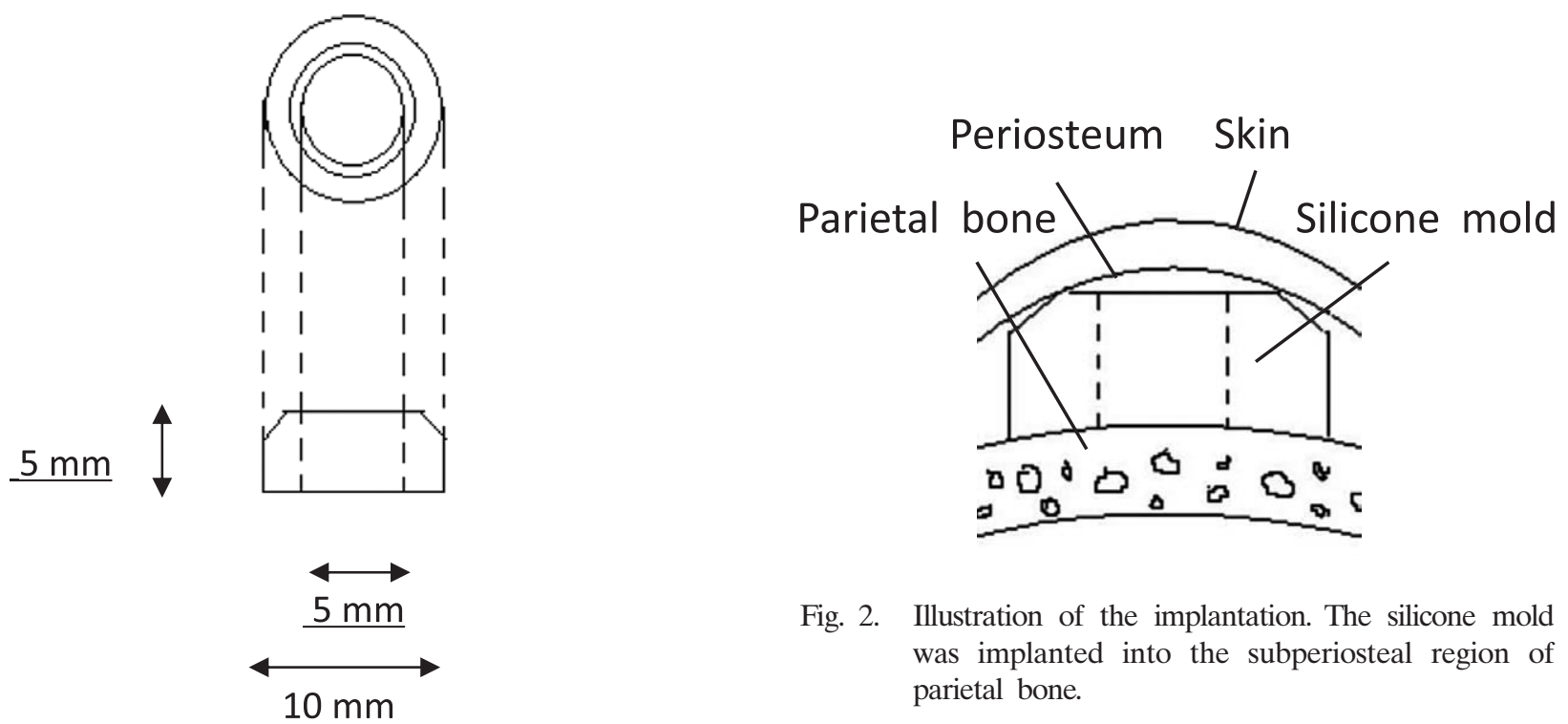

Fig. 2. Illustration of the implantation. The silicone mold was implanted into the subperiosteal region of parietal bone.

Fig. 1. Illustration of the silicone mold. It has a cylindrical shape with a $5 \mathrm{~mm}$ inner diameter, $10 \mathrm{~mm}$ outer diameter, and $5 \mathrm{~mm}$ height.

implanted into the subperiosteal pocket, and then the periosteum and skin were sutured with nylon thread in each layer (Fig. 2). The periosteal flap was sutured using single interrupted sutures and the skin flap was sutured tightly using a simple continuous suture. At 2, 4, and 8 weeks after surgery, rats were sacrificed and specimens (heads) were fixed in $10 \%$ neutral buffered formalin.

\section{Micro-CT Examination}

Before histological preparation, computerized X-ray tomography was performed with a microCT scanner (inspeXio SMX-90CT Micro Focus X-Ray CT System, Shimadzu, Kyoto, Japan). Whole head specimens were placed in the sample holder and scanned $(90 \mathrm{kV}, 110 \mu \mathrm{A})$. From the micro-CT scan datasets, three-dimensional models were built for visualization and morphological observation. Evaluation was performed in the sagittal plane at the area with the maximal diameter of the silicone mold.

The volume of newly formed bone in each silicone mold was measured with a threedimensional analyzing software (TRI/3D; RATOC, Tokyo, Japan). Bone density area inside the silicone mold was measured as the region of interest and evaluated as the bone volume. Values of bone volume in each group (2, 4, 8 weeks, $\mathrm{n}=5$ in all groups) were tested by oneway analysis of variance (Sheffe Test). Statistical analyses were carried out using IBM SPPS Statistics20 software (Japan IBM, Co., Tokyo, Japan). 


\section{Histological examination}

After the fixation in formalin, the silicone molds were removed. The specimens were decalcified by using Plank-Rychlo solution and embedded in paraffin. Sections at $5 \mu \mathrm{m}$ thickness were prepared and stained with hematoxylin and eosin, and then examined under the light microscope (ZEISS Axioskop 2 plus).

\section{Results}

\section{Macroscopic observation}

In the BMP-group at 8 weeks after surgery, cylindrical-shaped, newly induced bone was detected after removing the silicone mold. The bone was firmly adhered to the host parietal bone (Fig. 3). In the control group, no bone formation was detected. Soft connective tissue resembling a scar was observed in the silicone mold.

\section{Micro-CT scan analysis}

In the BMP-treated group, disc-shaped bone formation was observed adjacent to the periosteum at 2 weeks after surgery (Fig. 4A). At 4 weeks, new bone formation was observed adjacent to the original parietal bone, growing from the periosteal side and connecting to the parietal bone side (Fig. 4B). At 8 weeks, the inside of the silicone mold was almost filled with cylindrically shaped bone. The bone was composed of compact trabecular bone (Fig. 4C).

The three-dimensional measurement of volume revealed mean values for the newly formed bone at 2, 4, and 8 weeks of $4.15 \mathrm{~mm}^{3}$ (SD 4.12), $28.03 \mathrm{~mm}^{3}$ (SD 13.98), and $56.88 \mathrm{~mm}^{3}$ (SD 10.81), respectively (Fig. 5). Significant differences were recognized between 2 weeks and 4 weeks, and between 4 weeks and 8 weeks $(P<0.05)$. In the control group, bony tissue was not detected even at 8 weeks after surgery.

\section{Histological observation}

At 2 weeks after surgery, newly formed bone was observed in the subperiosteal region. The bone was characterized as woven bone (Fig. 6A, 6B). No apparent hematopoietic bone marrow

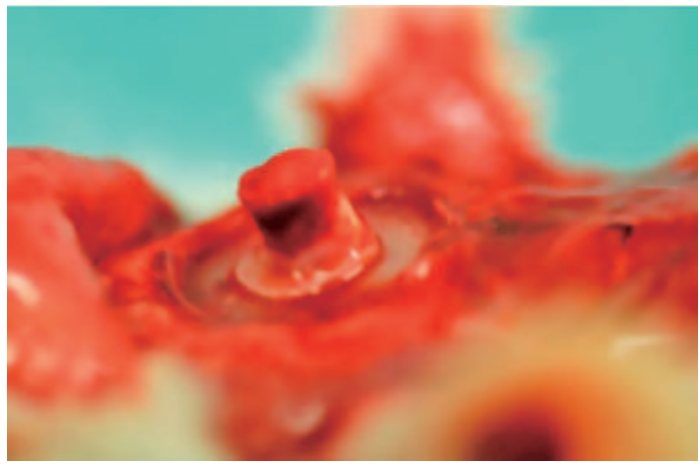

Fig. 3. Macroscopic findings at 8 weeks in the BMP-group. Bone formation was cylindrical shaped. 


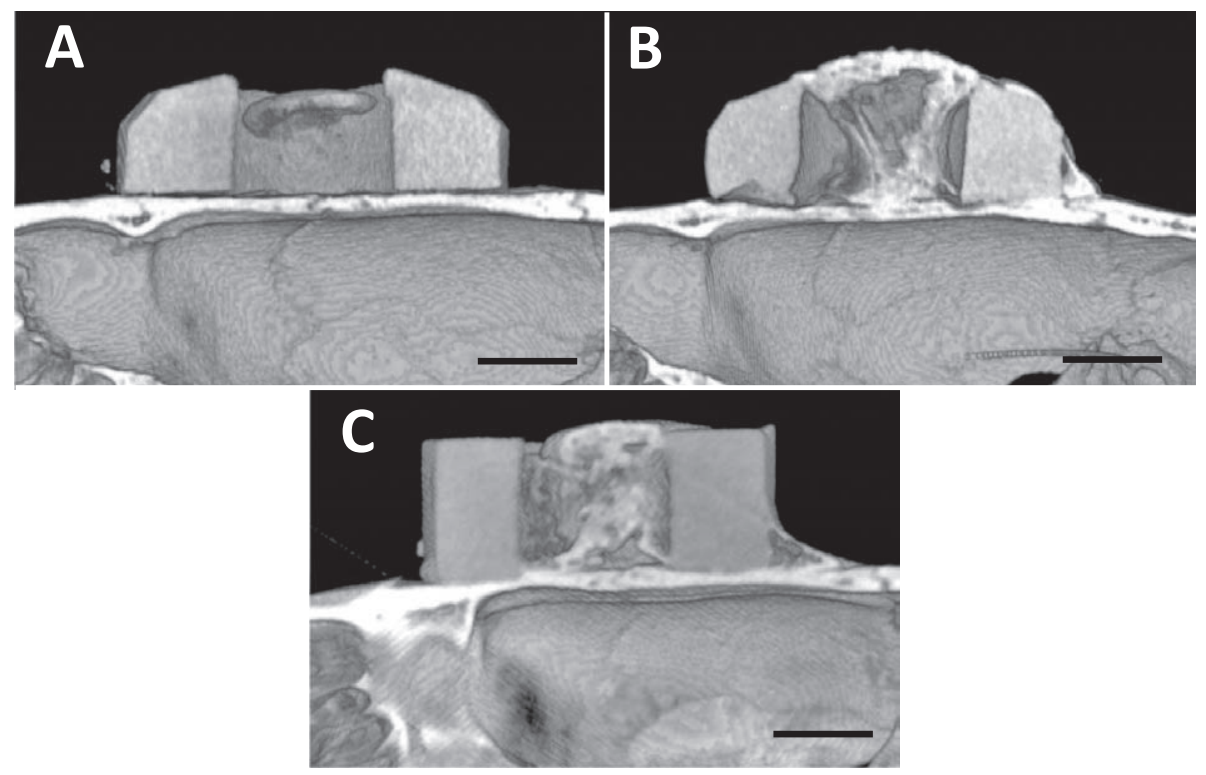

Fig. 4. Micro-CT finding in the BMP-group

A : 2 weeks. Disc-shaped bone formation was observed in the upper region of the silicone mold.

B : 4 weeks. Bone formation was observed in the region of the host parietal bone.

$\mathrm{C}$ : 8 weeks. Bone formation was observed in all regions of the silicone mold. Bar = $3 \mathrm{~mm}$.

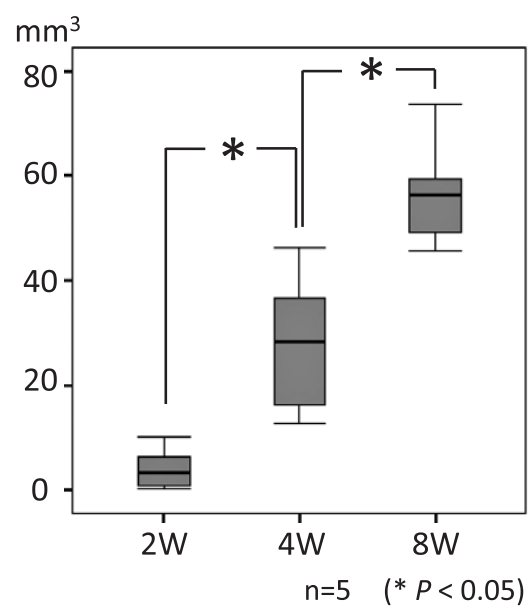

Fig. 5. Relationship between the newly formed bone volume and the weeks after implantation. Significant differences were recognized between $2 \mathrm{~W}$ and $4 \mathrm{~W}$, and between $4 \mathrm{~W}$ and $8 \mathrm{~W}$. (Columns : mean, bars : SD, ${ }^{*}: P<0.05$ )

was detected at this stage (Fig. 6B).

At 4 weeks, newly formed bone was observed adjacent to the periosteum and near the host parietal bone (Fig. 7A). This bone showed a trabecular structure, and osteocytes, located in the lacuna, were more obvious than those detected at 2 weeks (Fig. 7B). At this stage, the newly formed bone was not completely attached to the host parietal bone, however, many osteoblasts were observed along the surface between the host parietal bone and the newly formed bone 


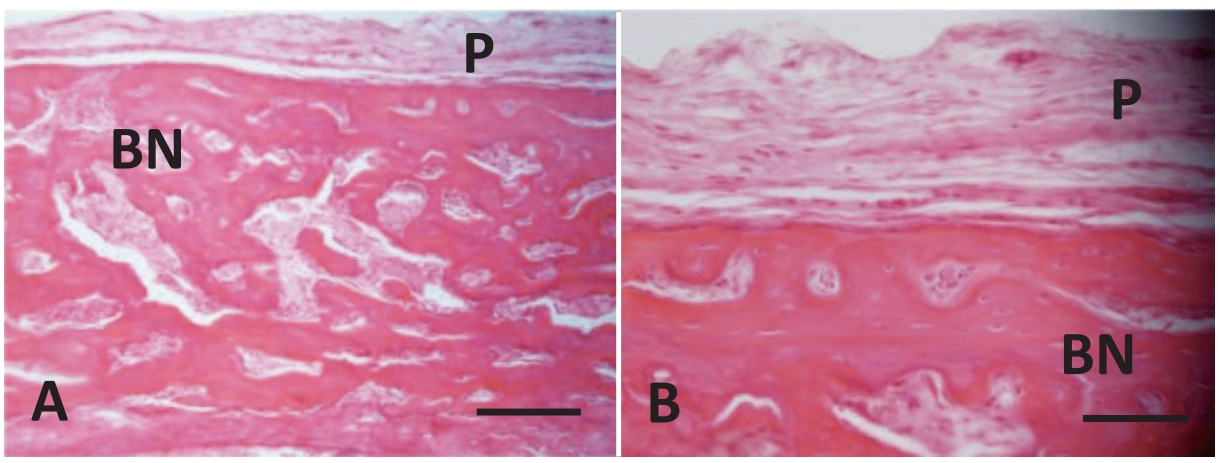

Fig. 6. Two weeks in the BMP-group

A : New bone formation was observed in the subperiosteal region. $\mathrm{P}$ : periosteum, $\mathrm{BN}$ : bone. Bar = $1 \mathrm{~mm}$.

B : Higher magnification of newly formed bone in Fig. 6A. Bar $=300 \mu \mathrm{m}$.

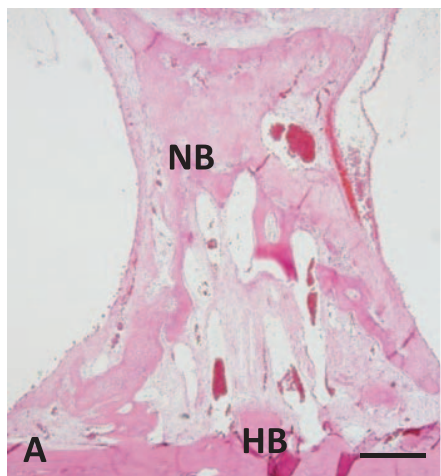

Fig. 7. Four weeks in the BMP-group

A : Periosteum, newly formed bone (NB), and host parietal bone $(\mathrm{HB})$ were seen. Bar $=800 \mu \mathrm{m}$.

$\mathrm{B}$ : Interface of the $\mathrm{HB}$ and NB. Many osteoblasts were observed lining the bone surface. Undifferentiated mesenchymal cells were also seen. Bar $=100 \mu \mathrm{m}$.

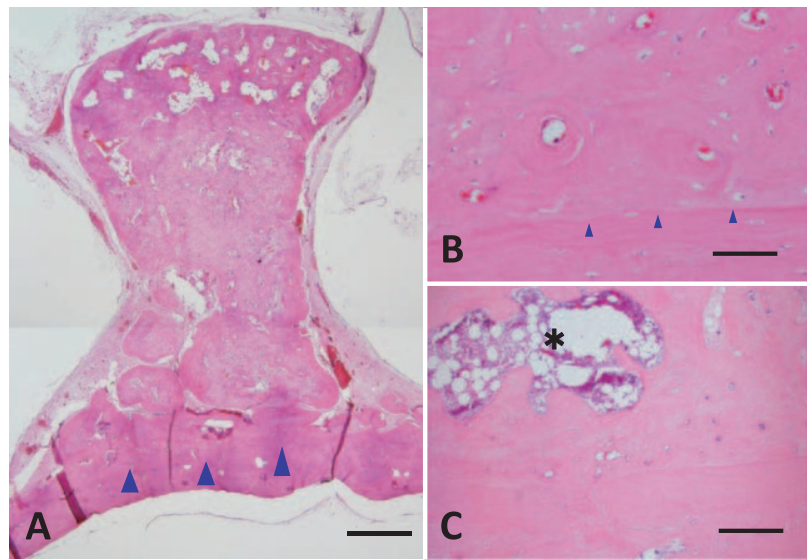

Fig. 8. Eight weeks in the BMP-group

A : The newly formed bone firmly adhered to the host parietal bone. Interface of the host parietal bone and newly formed bone (arrowheads), showing complete incorporation. Bar $=800 \mu \mathrm{m}$.

B: Higher magnification of the connecting region between the newly formed bone and parietal bone (arrowheads). Bar $=200 \mu \mathrm{m}$.

$\mathrm{C}$ : Bone marrow tissue (asterisk) was observed in this stage. Bar $=200 \mu \mathrm{m}$.

(Fig. 7B).

At 8 weeks, the newly formed bone showed a lamellar structure and it was firmly adhered to the host parietal bone (Fig. 8A, B). The connecting line between the newly formed bone and the host parietal bone was evident (Fig. 8B). Bone marrow was also distinctly present at this stage (Fig. 8C).

\section{Discussion}

Many kinds of materials have been used as the carrier for BMP-induced osteogenesis, such as hydroxyapatite $(\mathrm{HA})^{25)}, \beta$-tricalcium phosphate $(\beta \text {-TCP })^{26)}$, and atelocollagen ${ }^{19-22)}$. In this 
study, we selected atelocollagen sponge as a carrier because it is absorbable and it is approved for clinical adaptation ${ }^{20)}$.

One of the most important clinical objectives is to induce new bone with a definite and desirable shape. Alam et $a l^{23}$ reported that replacement and incubation of muscle tissue with a vascularized flap inside a BMP-containing silicone mold in the thigh of rat induced ectopic bone formation in the mold. Similarly, Hosoya et $a l^{24}$ induced bone formation in the silicone mold with a vascularized flap from fat tissue instead of muscle tissue, and the newly formed bone replicated the shape of the silicone mold. We modelled our present study on the same principle to form the desired shape of newly induced bone.

We further attempted to induce the bone by means of "onlay grafting" to the intact bone surface to simulate alveolar ridge bone augmentation without introducing a bone defect. Murata et $a l^{21)}$ reported that onlay augmentation using rhBMP-2 is much more difficult than inlay grafting, and attributed that to mechanical stress caused by the covering soft tissues preventing the volume and the shape of the newly formed bone to be achieved at the site of onlay grafting. We instead proposed that silicone molds have the required properties to resist such excessive mechanical stress.

In this study, the newly formed bone was first detected adjacent to the periosteum, and then was further formed at the region of the host parietal bone. Adeyemo et $a l^{22}$ indicated that osteoclasts were predominantly located at the graft-host interface in the early stage of onlay grafting in sheep mandible, and thus suggested that osteoclastic bone resorption on the host bone surface might be necessary when new bone formation is induced by BMP. In this study, we could not detect active bone resorption in the detected osteoclasts during the experimental period.

Osteogenic cells have been identified in the periosteum ${ }^{27-29)}$, whereas few of these cells would be located on parietal bone due to the removal of the periosteum during the surgical operation. This outcome might explain the difference in initiation of the new bone formation between the two regions.

It was suggested that the atelocollagen sponge acted as a carrier of BMP and "biological scaffold" for osteoinduction, and that the silicone mold played the role of a "physical scaffold" to induce the desired bone shape. It was also reported that ectopic newly formed bone induced by rhBMP-2 in rat thigh was intact without resorption in the silicone molds up to 48 weeks ${ }^{24)}$. In this study, active hematopoietic bone marrow was induced inside the newly formed bone in the silicone mold, indicating the maturation of bone.

Silicone molds are useful for maintaining induced bone; however, the mold should be removed after bone augmentation and before the installation of the dental implants, prompting the question as to whether the shape and volume of the augmented bone would be maintained after removal of the silicone mold. Further investigation is needed to establish effective clinical methods for applying BMP-induced bone formation.

In conclusion, newly formed bone could be induced in the parietal subperiosteal region of rats using rhBMP-2 with an atelocollagen sponge within a silicone mold. The new bone replicated 
the shape of the silicone mold and was firmly adhered to the host bone.

\section{Acknowledgements}

This study was supported by a grant from the Japan Society of the Promotion of Science (No. 20592149, No. 3592999).

\section{Conflict of interest disclosure}

The authors declare that there are no conflicts of interest.

\section{References}

1) Momtaheni DM, Schweitzer K, Muenchinger F. Technique for stabilization of autogenous cancellous bone grafts in sinus lift procedures. Oral Surg Oral Med Oral Pathol. 1994;78:14-16.

2) Dario LJ, English R Jr. Chin bone harvesting for autogenous grafting in the maxillary sinus: a clinical report. Pract Periodontics Aesthet Dent. 1994;6:87-91.

3) Krekmanov L, Heimdahl A. Bone grafting to the maxillary sinus from the lateral side of the mandible. $\mathrm{Br} J$ Oral Maxillofac Surg. 2000;38:617-619.

4) Triplett RG, Schow SR. Autologous bone grafts and endosseous implants: complementary techniques. J Oral Maxillofac Surg. 1996;54:486-494.

5) Garg AK. Augmentation grafting of the maxillary sinus for placement of dental implants: anatomy, physiology, and procedures. Implant Dent. 1999;8:36-46.

6) Steinberg B, Padwa BL, Boyne P, et al. State of the art in oral and maxillofacial surgery: treatment of maxillary hypoplasia and anterior palatal and alveolar clefts. Cleft Palate Craniofac J. 1999;36:283-291.

7) Auleda J, Bianchi A, Tibau R, et al. Hernia through iliac crest defects. A report of four cases. Int Orthop. 1995;19:367-369.

8) Ahlmann E, Patzakis M, Roidis N, et al. Comparison of anterior and posterior iliac crest bone grafts in terms of harvest-site morbidity and functional outcomes. J Bone Joint Surg Am. 2002;84-A:716-720.

9) Kolomvos N, Iatrou I, Theologie-Lygidakis $\mathrm{N}$, et al. Iliac crest morbidity following maxillofacial bone grafting in children: a clinical and radiographic prospective study. J Craniomaxillofac Surg. 2010;38:293-302.

10) Noia CF, Ortega-Lopes R, Olate $\mathrm{S}$, et al. Prospective clinical assessment of morbidity after chin bone harvest. $J$ Craniofac Surg. 2011;22:2195-2198.

11) Kent JN, Quinn JH, Zide MF, et al. Alveolar ridge aygmentation using nonresorbable hydroxylapatite with or without autogenous cancellous bone. J Oral Maxillofac Surg. 1983;41:629-642.

12) Block MS, Kent JN. Long-term radiographic evaluation of hydroxylapatite-augmented mandibular alveolar ridges. $J$ Oral Maxillofac Surg. 1984;42:793-796.

13) Rothstein SS, Paris DA, Zacek MP. Use of hydroxylapatite for the augmentation of deficient alveolar ridges. $J$ Oral Maxillofac Surg. 1984;42:224-230.

14) Barkhordar RA, Meyer JR. Histologic evaluation of a human periapical defect after implantation with tricalcium phosphate. Oral Surg Oral Med Oral Pathol. 1986;61:201-206.

15) Piche JE, Graves DT. Study of the growth factor requirements of human bone-derived cells: a comparison with human fibroblasts. Bone. 1989;10:131-138.

16) Urist MR. Bone: formation by autoinduction. Science. 1965;150:893-899.

17) Wozney JM, Rosen V, Celeste AJ, et al. Novel regulators of bone formation: Molecular clones and activities. Science. 1988;242:1528-1534.

18) Wang EA, Rosen V, D'Alessandro JS, et al. Recombinant human bone morphogenetic protein induces bone for- 
mation. Proc Natl Acad Sci U S A. 1990;87:2220-2224.

19) Fujimura K, Bessho K, Kusumoto K, et al. Experimental studies on bone inducing activity of composites of atelopeptide type I collagen as a carrier for ectopic osteoinduction by rhBMP-2. Biochem Biophys Res Comm. 1995;208:316-322.

20) Kaihara S, Bessho K, Fujimura K, et al. Experimental study of bone inducing ability of recombinant human bone morphogenetic protein-2 with atelo-collagen sponge (TERUPLUG【R】) as a carrier: comparison with atelopeptide collagen (Cellmatrix LA 【R】) as a carrier. Jpn J Oral Maxillofac Surg. 2003;49:241-2445. (in Japanaese).

21) Murata M, Huang BZ, Shibata T, et al. Bone augmentation by recombinant human BMP-2 and collagen on adult rat parietal bone. Int J Oral Maxillofac Surg. 1999;28:232-237.

22) Adeyemo WL, Reuther T, Bloch W, et al. Healing of onlay mandibular bone grafts covered with collagen membrane or bovine bone substitutes: a microscopical and immunohistochemical study in the sheep. Int J Oral Maxillofac Surg. 2008;37:651-659.

23) Alam MI, Asahina I, Seto I, et al. Prefabricated vascularized bone flap: a tissue transformation technique for bone reconstruction. Plast Reconstr Surg. 2001;108:952-958.

24) Hosoya M, Maruoka Y, Oda M, et al. Bone with a vascular flap induced from fat tissue with the use of rhBMP-2 in rats. $J$ Dent Res. 2003;82:581-584.

25) Ono I, Ohura T, Murata M, et al. A study on bone induction in hydroxyapatite combined with bone morphogenetic protein. Plast Reconstr Surg. 1992;90:870-879.

26) Breitbart AS, Staffenberg DA, Thorne $\mathrm{CH}$, et al. Tricalcium phosphate and osteogenin: a bioactive onlay bone graft substitute. Plast Reconstr Surg. 1995;96:699-708.

27) Nakahara H, Bruder SP, Goldberg VM, et al. In vivo osteochondrogenic potential of cultured cells derived from the periosteum. Clin Orthop Relat Res. 1990;(259):223-232.

28) Heino TJ, Hentunen TA. Differentiation of osteoblasts and osteocytes from mesenchymal stem cells. Curr Stem Cell Res Ther. 2008;3:131-145.

29) Colnot C. Skeletal cell fate decisions within periosteum and bone marrow during bone regeneration. J Bone Miner Res. 2009;24:274-282.

[Received June 8, 2017 : Accepted September 19, 2017] 